\title{
HACIA LA TRANSFORMACIÓN DE LA CULTURA ORGANIZACIONAL DE UNA AGENCIA PÚBLICA: UN MODELO HÍBRIDO DE INTERVENCIÓN
}

\author{
Blanca Ortiz-Torres ${ }^{1}$ \\ Wilmarie Santiago-López \\ Liz M. Cruz-Miranda \\ Yamilé Núñez-Maldonado \\ Tamara Acosta Ramírez \\ Universidad de Puerto Rico, Recinto de Río Piedras, Puerto Rico
}

\section{RESUMEN}

Uno de los niveles de intervención de la Psicología Comunitaria (PC) es el nivel organizacional. La intervención descrita es sintónica con los valores y principios de la PC. Los objetivos del proyecto son: aquilatar (assess) los elementos de la cultura organizacional de una dependencia gubernamental en Puerto Rico que constituyen fortalezas y debilidades para el logro de su misión y desarrollar acciones colectivas específicas para motivar y potenciar las capacidades de todos los sectores de la organización para que se involucren en procesos de cambio que aumenten la eficiencia y la efectividad de la agencia, así como la satisfacción individual y colectiva de empleados/as a todos los niveles. El proyecto fue desarrollado en dos fases: una de investigación y otra de intervención. Todos los sectores de la organización participaron en el diseño y ofrecieron sugerencias sobre su alcance e implantación. La fase de intervención sigue un Modelo de Competencias que permite describir el conocimiento, las destrezas y el comportamiento necesario para el desempeño efectivo en una organización. Desarrollamos cinco módulos de capacitación y se administraron pre y post pruebas antes y después de la implantación de los módulos de capacitación. La intervención permitió confirmar la posibilidad de cambio organizacional con implicaciones para el cambio individual.

\section{Palabras claves}

cultura organizacional, Investigación-Acción-Participativa, cambio organizacional

\begin{abstract}
One of the intervention levels of Community Psychology (PC) is the organizational level. The intervention described is syntonic with the values and principles of the PC. The objectives of the project are: assess the elements of the organizational culture of a government agency in Puerto Rico that constitute strengths and weaknesses for the achievement of its mission and develop specific collective actions to motivate and enhance the capacities of all sectors of the organization to get involved in processes of change that increase the efficiency and effectiveness of the agency, as well as the individual and collective satisfaction of employees at all levels. The project was developed in two phases: one for research and one for intervention. All sectors of the organization participated in the design and offered suggestions on its scope and implementation. The intervention phase follows a Competency Model that allows describing the necessary knowledge, skills and behavior for effective performance in an organization. We developed five training modules and pre and post tests were administered before and after the implementation of the training modules. The intervention confirmed the possibility of organizational change with implications for individual change.
\end{abstract}

\section{Keywords}

organizational culture, Participatory Action Research, organizational change

Correspondence about this article should be addressed to Blanca Ortiz-Torres. Email: b.ortiz@upr.edu 


\section{TOWARDS THE TRANSFORMATION OF THE ORGANIZATIONAL CULTURE OF A PUBLIC AGENCY: A HYBRID} INTERVENTION MODEL

Uno de los niveles de intervención de la Psicología Comunitaria (PC) es el nivel organizacional (Resto Olivo, Torres-López, \& Serrano-García, 2006). Algunos de los supuestos que guían las intervenciones a este nivel desde la disciplina son: (1) la transformación de la organización como sistema y no necesariamente de los individuos que la componen; (2) la organización es un sistema complejo que debe ser estudiado si se quiere entender el comportamiento individual que se da dentro de la misma; (3) la organización es un proceso más que una estructura (cambios en una parte de la organización producen cambios en otras). Nuestro trabajo estuvo guiado, además de por estos supuestos, por los valores y principios de la PC, particularmente el acceso a recursos y la transformación de relaciones asimétricas de poder para promover relaciones más equitativas en un escenario de trabajo.

La cultura organizacional tiene una importancia medular para alcanzar las metas de una organización (Schein, 1985); de hecho, algunos/as se refieren a la cultura organizacional como el clima y las prácticas que las organizaciones desarrollan para cumplir con sus objetivos y valores. Schein (1985) ha definido la cultura organizacional como:

Patrón de supuestos (assumptions) que un grupo determinado ha inventado, descubierto o desarrollado para lidiar con sus problemas de adaptación externa e integración interna y que, han funcionado de tal manera que se les enseña a nuevos miembros como si fuera la forma correcta de percibir, pensar y sentir en relación con esos problemas" (p. 3).

Forman parte de esta cultura, también, el aprendizaje compartido y acumulado en la organización que incluye elementos conductuales, emocionales y cognitivos, que provocan la socialización de sus miembros para alcanzar estabilidad y consistencia.

La cultura organizacional según Hemmelgarn, Glisson y James (2006) es "la forma en que se hacen las cosas aquî". Esa "forma de hacer las cosas" afecta la naturaleza de los servicios que provee una organización. Para Bolman y Deal ( 2017) la cultura organizacional es tanto un producto como un proceso. La cultura como producto, cristaliza el conocimiento acumulado de la experiencia mientras que, como proceso, se renueva y se recrea a medida que los nuevos miembros aprenden las viejas formas y, finalmente, se convierten en maestros. Igualmente, la cultura organizacional incorpora varios elementos que, en conjunto, reflejan una particular identidad de una organización, que es aprendida, internalizada y practicada por sus miembros.

Aunque no existe consenso sobre el particular, se reconocen diferencias en la cultura del sector público cuando se compara con el sector privado. De acuerdo con Suárez Pachón, Parra Triviño, Herrera Quintana y Peralta Gómez (2013), las organizaciones privadas están orientadas a los resultados y a sistemas abiertos, predominando la comunicación efectiva y las acciones colectivas. Por su parte, las organizaciones públicas se orientan más a los empleados/as y a los sistemas autoritarios, destacándose el departamento de recursos humanos en el control de los procesos. Estudios en empresas públicas han revelado que su cultura se ve afectada por culturas políticas predominantes. También se ha encontrado que se relaciona directamente la antigüedad con poder y liderazgo, lo que puede provocar que los/as empleados nuevos/as no expresen sus sentimientos y emociones para no ser calificados/as como desafiantes. Los autores señalan que algunos estudios han concluido que es difícil lograr cambiar la cultura de una institución pública, entre otras cosas, por la jerarquía vertical y la poca tolerancia a la frustración, provocando miedo colectivo y dificultando la innovación.

Cameron (2008) señala que la cultura organizacional puede ser indetectable la mayor parte del tiempo porque no se articula ni se desafía abierta o intencionalmente. Con frecuencia, muchos de los miembros de una organización desconocen su cultura hasta que ésta es cuestionada, hasta que se experimenta una nueva cultura, o hasta que la cultura se hace abierta y explícita a través de un marco o modelo. Este autor asegura que cambiar la cultura de una organización es un objetivo muy difícil de lograr, no solo porque la cultura tiende a no reconocerse, sino porque una vez que se establecen las interpretaciones, los valores y los patrones comúnmente compartidos son difíciles de modificar. Sin embargo, una vez se ha determinado que el cambio cultural es un objetivo deseado, los miembros de una 
organización pueden participar en una serie de acciones que pondrán en movimiento un proceso de cambio cultural. La cultura organizacional se puede constituir y transformar tanto en una organización como en una unidad de esta (Tsai, 2011).

Nuestro equipo de investigación recibió el acercamiento inicial para desarrollar este proyecto por parte del sindicato que representa a la mayoría de empleados/as de una agencia gubernamental en Puerto Rico. A este grupo les parecía que la organización no cumplía con su misión, estaba altamente politizada y no ofrecía sus servicios eficientemente. Junto con miembros del sindicato trabajamos una propuesta que luego fue presentada y discutida con los cuatro sectores principales que constituyen la organización (id est, empleados/as de conservación y mantenimiento, empleados/as de oficina, gerenciales y la administración central). Luego de recibir recomendaciones sobre el proyecto propuesto, los cuatro sectores articularon un acuerdo para que la investigación se realizara, por ser de interés para todos.

Nos enfocamos en la cultura organizacional considerando que cada organización tiene sus prácticas, procesos, lenguajes, códigos de vestimenta, entre otros elementos que la distinguen. Los objetivos de la investigación eran: aquilatar (assess) los elementos de la cultura organizacional de una dependencia gubernamental que constituyen fortalezas y debilidades para el logro de su misión y desarrollar acciones colectivas específicas para motivar y potenciar las capacidades de todos los sectores de la organización para que se involucren en procesos de cambio que aumenten la eficiencia y la efectividad de la agencia, así como la satisfacción individual y colectiva de empleados/as a todos los niveles. Durante la fase de investigación así como en la de intervención sostuvimos reuniones periódicas con los/as representantes designados/as por cada sector para discutir la evolución del proyecto y recibir insumo sobre diversas dimensiones del trabajo.

\section{Método}

Coincidimos con Fals Borda (1992), cuando advierte que la producción del conocimiento no es neutral; siempre responde a la situación y a los intereses de los sujetos que lo producen desde su base social. De ahí que tanto la fase de investigación como la de intervención se han desarrollado siguiendo el modelo de Investigación Acción Participativa (IAP). El modelo permite recoger y analizar información y actuar sobre los problemas con el propósito de encontrarles soluciones y promover la transformación en un grupo, comunidad u organización, siempre en consulta con las personas afectadas o que son sujetos de la investigación que se desarrolla (Balcázar, 2003). Para la investigación se emplearon las técnicas de análisis de documentos, entrevistas a profundidad a informantes claves, grupos focales y encuestas a clientes. A continuación describimos la fase de investigación.

\section{La investigación}

Seleccionamos la IAP como método porque permite problematizar la situación planteada y descubrir las contradicciones existentes y los posibles marcos de acción ante el fenómeno de estudio. La investigación participativa nos permitió lograr que todos los sectores que componen la organización, i.e. empleados/as de mantenimiento, profesionales y gerenciales, pudiesen comprender la complejidad de su centro de trabajo. Desde este modelo, como investigadoras buscamos consistentemente apoyarles en la identificación, problematización y comprensión de la cultura de su organización y la posibilidad de transformarla. Se esperaba que el cambio se concretara en modificaciones en las formas de relacionarse y ejercer liderazgo, en una mayor participación en procesos decisionales de la corporación, en un aumento en la eficacia y la eficiencia de la misma y en una mayor rendición de cuentas.

Comenzamos con la problematización de la cultura organizacional de la entidad gubernamental. Este proceso consistió en elegir una muestra de informantes claves que, por su gran conocimiento de la dependencia o por el cargo que ocupan o han ocupado proveyeron información valiosa sobre las limitaciones y fortalezas tanto de la estructura como de la cultura de la corporación pública en entrevistas individuales. Se entrevistó a profundidad a ocho informantes claves que representaban a los diversos 
sectores de la organización. La entrevista seguía unas preguntas guías, aunque se añadieron preguntas en aquellos casos en que era pertinente. De estas entrevistas emergieron temas y asuntos que permitieron diseñar grupos focales en los que participó personal gerencial, profesional y de mantenimiento.

Como es característico de la IAP, se estableció un diálogo crítico entre el saber académico y el conocimiento de los trabajadores/as dirigido hacia la transformación necesaria para lograr la eficiencia y la eficacia de la organización. Reconociendo que la realidad responde a la situación y a los intereses de los sujetos, nuevas "definiciones" surgieron para ir apuntando hacia lo que sería el cambio en cultura organizacional. Para llevar a cabo esta tarea, desde los grupos focales nos acercamos no sólo al conocimiento empírico sino desde el sentido común y conocimiento práctico de la base. Se celebraron tres grupos focales: dos con una muestra de empleados/as de conservación, mantenimiento y oficinistas y otro con empleados/as gerenciales. Los/as participantes en los grupos focales se seleccionaron al azar, del listado de empleados/as que nos suministró la organización. Se seleccionó un total de 20-25 participantes para cada grupo; escogiendo la quinta persona en la lista y luego cada cinco personas. Participó un total de 40 personas en los tres grupos. El contenido de las entrevistas y los grupo focales fue analizado usando la técnica de análisis de contenido para desarrollar categorías y subcategorías de análisis.

Además, se diseñó y se administró una encuesta al cliente (en el caso de esta corporación pública los clientes son otras dependencias gubernamentales), que permitió tener la mirada externa de la organización. La encuesta iba dirigida a conocer la satisfacción de los clientes con la calidad de los servicios que ofrece la corporación, con el tiempo con el que se atienden sus solicitudes y el trabajo que realizan sus empleados/as. Los niveles de satisfacción se evaluaban en una escala de cuatro puntos, desde "muy insatisfecho" hasta "muy satisfecho". También se solicitaban sugerencias para mejorar la calidad del servicio ofrecido. Los clientes se seleccionaron al azar de dos listas suministradas por la agencia. Para cada región del país a la que la organización ofrece servicio se seleccionó un 15\% del total de clientes. Fue necesario sustituir clientes por diversas razones: 1) no hubo respuesta a las llamadas realizadas luego de un promedio de cinco intentos; 2) el funcionario/a a cargo indicó que la agencia o escuela no pertenece a la región bajo la que aparece en la lista; 3) el personal contactado en la agencia se negó a participar en la entrevista; 4) indicaron que el funcionario/a a cargo no estaba disponible; y 5) indicaron que no estban autorizados/as para conceder la entrevista. De las 25 entrevistas esperadas, se completaron 17.

A través de la investigación participativa se generó un proceso crítico, mediante el cual los trabajadores/as pudieron identificar sus roles y participar en acciones dirigidas hacia el cambio institucional, comenzando a poner en práctica iniciativas dirigidas a lograr dicho cambio, sin la necesidad de esperar por instrucciones de la alta gerencia. A estas iniciativas le llamamos 'acciones transformadoras'. Al finalizar la fase de investigación, el equipo de investigación hizo la correspondiente devolución de los datos que sirvió para que los empleados/as se apropiaran aún más del producto de la investigación/acción. Los hallazgos se discutieron en reuniones con cada sector, se contestaron preguntas, se amplió la interpretación de los hallazgos y se trabajaron en recomendaciones para la fase de intervención, que describimos a continuación.

\section{La intervención}

La fase de intervención se desarrolló a partir de los hallazgos obtenidos en la fase de investigación y guiada por el Modelo de Competencias (Cordero-Nieves et al., 2014) que permite describir el conocimiento, las destrezas y el comportamiento necesario para el desempeño efectivo en una organización. A partir de las competencias descritas por Cordero-Nieves y colaboradores/as y de las discusiones sostenidas con los actores descritos/as, se desarrollaron cinco módulos de capacitación para cada criterio: liderazgo, empoderamiento, ética, innovación y rendición de cuentas. El diseño de los módulos siguió el Modelo Secuencial-Reiterativo (SIM) de Milano y Ullius (1998). En cada módulo se incluyó una serie de actividades que refuerzan los principios de la andragogía o aprendizaje en adultos. Se consideró pertinente diseñar técnicas prácticas de aprendizaje para cumplir con estos principios y 
facilitar un análisis más profundo de la cultura organizacional actual y aquella a la que aspiran. Se administraron pruebas antes y después de la implantación de los módulos de capacitación.

El módulo de liderazgo incluía las competencias de trabajo en equipo, creatividad, aceptación al cambio y la flexibilidad, innovación, comunicación efectiva, relaciones interpersonales, planificación estratégica y compromiso. Los ejercicios diseñados giraban alrededor del trabajo en subgrupos para discutir, actuar o diseñar una tarea. Luego discutían los resultados y analizaban cómo se relacionaba a los temas del módulo. Uno de los ejercicios era el de la caja de herramientas. Cada participante tenía que identificar la herramienta más utilizada en su trabajo y discutir qué competencias u otras herramientas consideraba necesarias e imprescindibles para realizar eficientemente su trabajo. Al final de cada módulo los/as participantes tenían que llevarse una herramienta (i.e. competencia) que deseaban mejorar o utilizar para su trabajo. En el próximo módulo se discutía el progreso de cada cual con la competencia identificada.

Para el segundo y tercer módulo, los de Empoderamiento y Ética, se incorporaron las competencias de iniciativa, toma de decisiones participativa, enfrentar riesgos, liderazgo ético y dilemas éticos. El trabajo con estas competencias incluía ejercicios grupales y discusión de videos. Al final, los/as participantes discutían un caso asignado y señalaban principios éticos que fueron violados.

Con los módulos de Rendición de Cuentas e Innovación se cerraban las sesiones de adiestramiento. Estos módulos trabajaban dos competencias que fueron muy bien recibidas por los/as participantes: desarrollo de proyectos pilotos y establecimiento de metas organizacionales claras. Los/as participantes compartieron anécdotas sobre proyectos pilotos que se han realizado en ciertas regiones y lo exitosos que han sido. En el ejercicio de las metas claras, los/as participantes discutieron lo que entendían era la misión de la agencia. Los temas sobre el uso y abuso de la autoridad pública y la seguridad en el uso de los recursos públicos provocaron mucha discusión entre los/as participantes.

Durante los ejercicios y dinámicas analizábamos nuestras observaciones, las expresiones, conductas e interacciones de los/as participantes. De esta manera, la intervención era también una técnica de recopilación de información. Nuestras observaciones reforzaron los hallazgos de la primera fase que se limitó a un grupo pequeño de empleados/as, mostrando cierta consistencia. En términos generales, se observaba disgusto con la actual cultura organizacional de la organización, deseos de aprender y de transformar.

Con el objetivo de determinar si hubo cambio en las competencias incluidas en este modelo, luego de haber participado en los módulos de capacitación, desarrollamos instrumentos de pre y post prueba para cada uno. Cada prueba fue diseñada a partir de los comportamientos esperados en el modelo de competencias. Diseñamos pruebas de selección múltiple y situaciones hipotéticas. Los resultados cualitativos y cuantitativos de estas pruebas ofrecieron información valiosa sobre el inicio del proceso de cambio en las regiones, dirigidos a la transformación de la cultura organizacional de la agencia.

Desarrollamos e impartimos una sesión/módulo de adiestramiento a los/as delegados/as de los sindicatos. Las competencias en ese módulo incluían los siguientes criterios: trabajo en equipo, manejo de conflictos y manejo y expresión de emociones. La selección de estas competencias se basó en los hallazgos de la fase de investigación y en las conversaciones sostenidas con todos los sectores del personal de la agencia. De igual forma, para determinar los cambios de las competencias incluidas en este módulo, desarrollamos una pre y post prueba.

Además de la capacitación, aquilatamos los procesos administrativos de dos importantes sistemas de la corporación pública, con el fin de determinar si como resultado de la capacitación, se iniciaron acciones transformadoras para incidir en dichos procesos. El primero fue la evaluación de los procesos de compra y el segundo el sistema de servicio al cliente. El Sistema de Servicio al Cliente se encarga de almacenar los datos de los trabajos de inspección y reparación realizados y mantiene una comunicación con los clientes que reciben el servicio y la corporación, además de medir el nivel de satisfacción de los clientes luego de ofrecido el servicio. 
Se evaluaron dos tiempos, uno en marzo de 2016 y otro en octubre del mismo año. La primera fecha examina los procesos antes de que los/as participantes tomaran los talleres sobre las competencias mencionadas anteriormente y la segunda hace lo mismo una vez culminaron los talleres. Se estudió el tiempo de espera para gestionar una petición de compras de un producto o servicio.

\section{Resultados}

De acuerdo con los/as participantes, la cultura organizacional de la agencia está caracterizada por: líneas de mando difusas, abandono del sistema de mérito, exagerada intervención de la política partidista y un exceso de burocracia con limitada participación de los sectores medios y bajos de la misma. En esa cultura están ausentes la capacitación y educación continua de los/as empleados/as. Se identifican como fortalezas el compromiso de empleados/as, técnicos bien preparados y proactivos/as y una gran amplitud de destrezas y servicios ofrecidos.

En la fase de intervención capacitamos a 167 supervisores/as. Luego de tomar el módulo de liderazgo, aumentaron principalmente las competencias de: promoción de la diversidad, búsqueda de recursos creativos, percepción de cambios en la agencia como posibilidad de nuevos aprendizajes, promoción de autonomía en empleados/as, desarrollo de habilidades en los miembros del equipo a través de un sistema de capacitación, entre otras. Luego del módulo de empoderamiento y ética, aumentaron principalmente las competencias de: reconocimiento de las iniciativas de los/as empleados/as, toma de decisiones participativas y discreción y lealtad a la organización.

Capacitamos a 40 delegados y líderes sindicales que, luego de asistir a los talleres, demostraron aumento en las competencias de: uso de mediación y de decisiones compartidas para solución de conflictos, evaluación de las repercusiones de las acciones que se toman en situaciones de frustración y negociación con los/as supervisores en situaciones de tensión o conflicto. Algunos/as participantes refirieron cómo los módulos de capacitación impactaron el trabajo y sus vidas, pues utilizaron herramientas de los talleres para solucionar situaciones familiares. A continuación, presentamos los resultados sobre las acciones transformadoras

\section{Acciones Transformadoras}

Como parte de las acciones transformadoras generadas en este proyecto, se realizó un análisis de los procesos administrativos de dos importantes sistemas. El primero fue la evaluación de los procesos de las peticiones de compra en donde se estudió el tiempo de espera para gestionar una petición de compras de un producto o servicio. Se evaluaron dos tiempos: antes y después de que los/as participantes tomaran los talleres de capacitación.

\section{Tabla 1}

Cantidad de peticiones de compra trabajadas durante marzo y octubre de 2016

\begin{tabular}{lll}
\hline Tiempo de espera & $\begin{array}{l}\text { Marzo 2016 } \\
\mathrm{n}=152\end{array}$ & $\begin{array}{l}\text { Octubre } 2016 \\
\mathrm{n}=217\end{array}$ \\
\hline $0-20$ & $61 \%$ & $57 \%$ \\
$21-40$ & $15 \%$ & $40 \%$ \\
$41-60$ & $5 \%$ & $3 \%$ \\
$61-80$ & $4 \%$ & - \\
$81-100$ & $5 \%$ & - \\
$101-120$ & $6 \%$ & - \\
$121-140$ & $3 \%$ & - \\
$141-160$ & $1 \%$ & - \\
\hline
\end{tabular}


Como se puede observar en la tabla 1, aún cuando se trabajaron más peticiones de compra durante el segundo periodo estudiado $(\mathrm{n}=217)$, se reflejó una reducción significativa del tiempo de espera para gestionar cada petición. El $97 \%$ de las peticiones se trabajaron en 40 días o menos. En el primer tiempo se logró gestionar 76\% de las peticiones durante el mismo periodo aun cuando se recibieron 65 peticiones menos. No podemos inferir que el resultado en el tiempo de espera cambió exclusivamente por la capacitación que recibieron los supervisores y personal de la corporación, pero podemos mencionar que éstos/as estaban más conscientes de las competencias trabajadas, lo que pudo resultar en un cambio de actitud, responsabilidad laboral y motivación hacia el trabajo.

El segundo sistema analizado fue la evaluación de los procesos del Sistema de Servicio al Cliente (vale señalar que en el caso de la dependencia gubernamental con la que trabajamos, la clientela la constituyen otras agencias gubernamentales). Una vez completado el servicio, las personas informan cuán satisfechos/as estuvieron luego de ofrecido el servicio. Estos datos también se analizaron para los meses de marzo y octubre de 2016.

La tabla 2 muestra la cantidad de trabajos realizados durante los meses señalados y el nivel de satisfacción expresado por los clientes.

Tabla 2

Nivel de satisfacción de clientes tras recibir servicios por parte de los trabajadores

\begin{tabular}{ccc}
\hline Mes/Año & Peticiones de trabajo & Nivel de satisfacción \\
\hline Marzo 2016 & 1.582 & $81 \%$ Excelente \\
& & $19 \%$ Bueno \\
Octubre 2016 & 946 & $94 \%$ Excelente \\
& & $4 \%$ Bueno \\
& $2 \%$ Regular \\
\hline
\end{tabular}

El nivel de satisfacción de los clientes con el servicio ofrecido por parte de los trabajadores/as es alto. Luego de la capacitación, una gran mayoría de los clientes (94\%) calificó el servicio como "Excelente", en comparación con el primer tiempo (81\%).

\section{Discusión}

Las dos fases del proyecto de investigación-acción presentado, sugieren la posibilidad de cambio organizacional con implicaciones para el cambio individual, partiendo de los supuestos y valores de la Psicología Comunitaria. Como se ha descrito, el acercamiento al equipo de investigación no surgió de la administración central de la dependencia gubernamental, sino de uno de los sindicatos que representa a la mayoría de los/as trabajadores/as de la misma. Este grupo organizó reuniones y discusiones con los demás sectores de la organización en las que se fue configurando el diseño de la investigación y más tarde el contenido y forma de la intervención. El equipo de investigación, sirvió de facilitador y educador en el proceso de iniciar un proyecto de cambio, potenciando la iniciativa y participación de los trabajadores/as de la corporación pública. La investigación participativa muestra su capacidad para contribuir en escenarios laborales ampliando sus marcos conceptuales y promoviendo la problematización y la adopción de posiciones críticas que promuevan el cambio institucional y social. Nos parece que este es un modelo híbrido que permite promover el cambio organizacional desde supuestos teóricos y metodológicos distintos.

No obstante, no podemos descartar que ciertos factores externos (exempli gratia, crisis fiscal que atraviesa el país, políticas públicas obsoletas, la política partidista y los cambios de administración, entre otros) pueden influir en que la cultura organizacional de la agencia se siga viendo afectada. Destacamos la apertura que tuvieron algunos/as de los participantes durante estas sesiones. Se mostraban deseosos/as de aprender y de transformar una agencia que, para la mayoría, es una pieza importante para el desarrollo 
económico del país. De igual manera, recalcaron la falta de capacitación que se les da a los/as servidores públicos.

Desafortunadamente, no se dieron las condiciones para poder evidenciar que en efecto se transformó la cultura organizacional de la agencia gubernamental. Sin embargo, la intervención sí permitió confirmar la posibilidad de cambio organizacional con implicaciones para el cambio individual. Evaluamos los resultados obtenidos en la intervención utilizando como guía los siete pasos del proceso para cambiar la cultura organizacional (Cameron, 2008), identificando algunos ejemplos de acciones que se han efectuado y demostrado que está en movimiento ese cambio. Estos pasos inician cambios en procesos, conversaciones, lenguaje, símbolos y valores individuales y organizacionales.

Los siete pasos son: 1) clarificar el significado (aclarar los elementos que no cambiarán y aquellos que sí lo harán); 2) identificar historias (identificar uno o dos incidentes o eventos positivos que ilustren los valores clave que caracterizarán la futura cultura de la organización); 3) determinar iniciativas estratégicas (acciones diseñadas para realizar cambios importantes que producirán cambios en la cultura); 4) identificar pequeñas victorias (acciones inmediatas que representan pequeños pasos hacia el cambio de cultura); 5) establecer métricas, medidas y metas (determinar los indicadores clave de éxito, qué medir, cómo medirlo y cuándo se notarán ciertos niveles de progreso); 6) comunicación y símbolos (comunicar ayuda a superar la resistencia y generar compromiso, mientras que identificar los símbolos que significan un nuevo futuro es una parte importante del cambio cultural); y 7) desarrollo de liderazgo (el cambio de cultura rara vez ocurre de forma aleatoria o inadvertida en las organizaciones por tanto se requieren líderes que dirijan el proceso consciente y sistemáticamente). Ninguno de estos pasos por sí solo asegura que se producirá un cambio cultural, pero en combinación crean un gran impulso hacia el cambio fundamental de la cultura en las organizaciones.

A continuación, expondremos algunos ejemplos de acciones que ya se han encaminado hacia el cambio en la cultura organizacional de esta agencia. El primer paso del proceso, clarificar el significado de los procesos susceptibles de cambio y aquellos que no lo son, se pudo observar en una discusión que se generó entre gerenciales sobre la misión actual de la corporación pública. En la conversación, los/as supervisores señalaron que la misión de la agencia debe ser revisada y actualizada ya que no se realizan muchos de los servicios incluidos en la misma. Muchas de las funciones originales de la agencia fueron transferidas a otras instancias. Argumentaron que para actualizar la misión es indispensable la participación de todos los sectores de la organización.

A raíz de los hallazgos de la fase de investigación y en discusión constante con los representantes de los sindicatos y la gerencia, se propuso la implementación de acciones dirigidas a transformar la cultura organizacional de la agencia. Una de las acciones propuestas fue la facilitación de talleres de capacitación para la gerencia utilizando un modelo de competencias. Una de las competencias centrales de este modelo es la de liderazgo. Al inicio de la capacitación la mayoría de los supervisores/as expresó que, efectivamente, ejercían competencias de liderazgo; luego de tomar el módulo de liderazgo estuvieron de acuerdo en que se puede ejercer el liderazgo de forma transformadora. En las discusiones se articularon las diferencias entre el liderazgo actual y el tipo de liderazgo que se desea alcanzar. Esta estrategia cumple con el séptimo paso del proceso, desarrollo de liderazgo, porque cada aspecto del proceso de cambio cultural necesita líderes que se apropien de su implementación exitosa

Otras recomendaciones que emergieron del proyecto son: revisar todas las políticas públicas que afectan las funciones y operaciones de la dependencia, desarrollar capacitación que responda a las preocupaciones expresadas por todos los grupos y priorizar en en el área de ética. Además, se generaron recomendaciones sobre los procesos de compra y servicio al cliente. Para evaluar si se generó algún cambio en los procesos, una vez se concluyó la serie de talleres en la fase de intervención, se volvieron a analizar datos de esta oficina. En comparación con los primeros datos analizados, se pudo registrar un cambio positivo en la eficiencia del servicio. Este logro puede clasificarse como el cuarto paso del proceso; una pequeña victoria que representa una tendencia hacia el cambio cultural.

Entre las recomendaciones presentadas se encuentran el cambio en los procesos administrativos y reglamentos que pueden resultar en burocracia excesiva y dilación de procesos. Además, se recomendó brindar la oportunidad de capacitar a los empleados/as en diversos procesos administrativos relacionados 
a su trabajo. Según Siliceo-Aguilar (2004), algunos de los principales beneficios de la capacitación son facilitar la asimilación e internalización de los valores, incrementar la productividad personal y grupal, mejorar la calidad del desempeño, reducir el ausentismo, promover y enriquecer la cultura organizacional, entre otros. Finalmente, se recomendó la realización de autoevaluaciones que pueden ayudar a aquilatar cuán eficientes y efectivos pueden ser los procesos administrativos que llevan a cabo para, entonces, realizar ajustes o modificaciones. Es importante señalar que, como ocurrió en todas las etapas de la investigación y la intervención, las recomendaciones, se recogieron en un informe final que fue presentado y avalado por todas las organizaciones gremiales y la administración central de la corporación pública.

El clima organizacional afecta la satisfacción de los clientes con los productos o servicios que ofrece la empresa. Estos generan una imagen subjetiva acerca de la calidad de lo que reciben, de allí derivan, en parte, su satisfacción, la cual surge como respuesta emocional al comparar lo recibido con lo esperado (Salanova \& Peiró, 2005). De esta manera, si el personal ha cuidado de la atención y de la calidad es probable que el cliente esté más satisfecho (Toro-Álvarez \& Sanín-Posada, 2013).

Este proyecto apunta a las posibilidades de transformación de escenarios de trabajo de forma participativa, incluyendo investigación/acción que valide los saberes de todos los sectores, particularmente el de los/as trabajadores/as. La Psicología Comunitaria nos ofrece el sustento teórico y metodológico para así hacerlo. 


\section{Referencias}

Balcázar, F. E. (2003). Investigación acción participativa (iap): Aspectos conceptuales y dificultades de implementación. Fundamentos en humanidades, IV (7-8), 59-77.

Bolman, L. G., \& Deal, T. E. (2017). In Reframing organizations: Artistry, choice, and leadership. San Francisco, CA: Jossey-Bass Publishers.

Borda, F. (1992). La ciencia y el pueblo: Nuevas reflexiones. In M. C. Salazar (Ed.), La investigaciónacción participativa. Inicios y desarrollos (pp. 65-84). Madrid, España: Editorial Popular, Quinto Centenario.

Cameron, K. (2008). A process for changing organizational culture. In T. G. Cummings (Ed.), Handbook of Organizational Development (pp. 429-445). Thousand Oaks, CA: Sage Publishing.

Cordero-Nieves, Y., Santana-Rabell, L., Negrón-Portillo, M., Ríos-González, P. N., Santiago-Rivera, C. A., Punsoda-Díaz, J. A., . . Sánchez-Lugo, J. (2014). Reforma Gubernamental: Un nuevo modelo organizativo para Puerto Rico. San Juan, PR: Editorial Cordillera.

Hemmelgarn, A. L., Glisson, C., \& James, L. R. (2006). Organizational culture and climate: Implications for services and interventions research. Clinical Psychology: Science and Practice,, 13(1), $73-$ 89.

Milano, M., \& Ullius, D. (1998). Designing powerful training: the sequential-iterative model. San Francisco: Jossey-Bass/Pfeiffer.

Resto Olivo, J., Torres-López, L., \& Serrano-García, I. (2006). La psicología social-comunitaria en Puerto Rico: treinta años de trayectoria. Revista Puertorriqueña de Psicología, 17(1), 421-459.

Salanova, M., \& Peiró, J. M. (2005). Linking Organizational Resources and Work Engagement to Employee Performance and Customer Loyalty: The Mediation of Service Climate. Journal of Applied Psychology, 90(6), 1217-1227.

Schein, E. (1985). Organizational Culture and Leadership. San Francisco, CA: Jossey-Bass Publishers.

Siliceo-Aguilar, A. (2004). Capacitación sistemática. En Capacitación y desarrollo de personal (4ta edició, p. 165).

Suárez Pachón, D., Parra Triviño, M., Herrera Quintana, A., \& Peralta Gómez, M. (2013). Cultura organizacional en una empresa estatal colombiana: estudio de caso. . Tesis Psicológica, 8(2), 3051.

Toro-Álvarez, F., \& Sanín-Posada, A. (2013). Gestión del clima organizacional: Intervenciones basadas en evidencias. Cinsel. doi:https://doi.org/10.21772/gco.ibe

Tsai, Y. (2011). Relationship between organizational culture, leadership behavior and job satisfaction. BMC Health Services Research, 11(1), 98-106. doi:10.1186/1472-6963-1198 